Gastroenterologe 2014 - 9:511-511

DOI 10.1007/s11377-014-0919-y

Online publiziert: 26. Oktober 2014

(c) Springer-Verlag Berlin Heidelberg 2014
H. Messmann ${ }^{1} \cdot$ C. Trautwein ${ }^{2}$

${ }^{1}$ III. Medizinische Klinik, Klinikum Augsburg

2 Medizinische Klinik III, Universitätsklinikum Aachen, AÖR, Aachen

\title{
Gastroenterologische Intensivmedizin
}

Betrachtet man die Anzahl der beteiligten Organe mit Komplikationen in der Intensivmedizin, so kommt der Gastroenterologie eine entscheidende Rolle in der Gestaltung individueller, auf den $\mathrm{Pa}-$ tienten bezogener, aber auch allgemeiner Konzepte in der modernen Intensivmedizin zu. Die Rolle der Gastroenterologie in der Intensivmedizin ist dabei schon lange nicht mehr auf die Behandlung der Leberzirrhose und ihrer Komplikationen oder der akuten Pankreatitis begrenzt, sondern beschäftigt sich mit Veränderungen, gastroenterologischen Organkomplikationen und Interaktionen, die im Rahmen intensivmedizinischer Behandlungen kritisch kranker Patienten auftreten können. Ein Schwerpunktheft kann natürlich nicht alle relevanten Themen behandeln. Wir haben daher fünf Themenschwerpunkte herausgegriffen, die aus unserer Sicht wichtige Probleme in der Behandlung unserer Patienten darstellen.

Im ersten Artikel beschäftigen sich Huber et al mit dem Krankheitsbild der akuten Pankreatitis. Hierbei geht es unter anderem um das Zeitfenster für Diagnostik und Therapie. Dabei spielen Prognosescores für die Einschätzung des Schweregrads der Erkrankung, sowie konkrete Handlungsanweisungen eine zentrale Rolle, um die Patienten optimal zu therapieren.

Im Artikel von Bittinger et al wird das Thema der gastrointestinalen Motilitätsstörungen bei Intensivpatienten aufgegriffen. Diese Problematik spielt bei vielen Intensivpatienten fächerübergreifend eine große Rolle und ist daher eine häufige Konsilanforderung an den Gastroenterologen. Eine besondere Bedeutung spielen dabei Magenentleerungsverzögerungen, Diarrhoen und Obstipationen. Die Auto- ren zeigen dabei systematisch auf, welche Ursachen und therapeutische Möglichkeiten für die Patienten bestehen.

Der Beitrag von Braun et al hat als Thema die medikamentöse Stressulkusprophylaxe. Diese Therapie wird regelmäßig auf konservativen und operativen Intensivstationen eingesetzt. Da Blutungen des oberen Gastrointestinaltrakts nicht häufig sind, wird genauer auf die Indikation zur Therapie eingegangen, um mögliche Nebenwirkungen der Therapie zu vermeiden. Es wird dabei auf die Wertigkeit der Protonenpumpenhemmer und $\mathrm{H}_{2}$-Rezeptor-Antagonisten eingegangen, aber auch die Option der frühen enteralen Ernährung diskutiert.

Das akute Leberversagen stellt eine seltene aber prognostisch problematische Erkrankung dar. Deshalb sollten die Patienten frühzeitig in ein Transplantationszentrum verlegt werden, um alle therapeutischen Optionen inklusive der Lebertransplantation nutzen zu können. Der Artikel von Canbay et al beschäftigt sich bei diesem Krankheitsbild gezielt mit der Wertigkeit der Prognoseabschätzung durch Minilaparoskopie und Leberpunktion, um den Patienten in dieser lebensbedrohlichen Situation die richtige Therapie zukommen zu lassen.

Patienten mit Leberzirrhose haben auf der Intensivstation eine eingeschränkte Prognose. Ursachen dafür sind die typischen, lebensbedrohlichen Komplikationen der Leberzirrhose. Durch ein strukturiertes intensivmedizinisches Management kann jedoch die Prognose entscheidend verbessert werden. Im Artikel von Tacke et al wird daher auf die unterschiedlichen Optionen eingegangen, die abhängig von der jeweiligen Komplikation aktuell zur Verfügung stehen.
Wir hoffen, dass wir Ihnen mit dieser Auswahl einen guten Überblick über wichtige Themengebiete der gastroenterologischen Intensivmedizin vermitteln können und dass Sie dadurch viele praktische Hinweise für Ihre tägliche Praxis erhalten.

\section{Mit herzlichen Grüßen}

Ihre

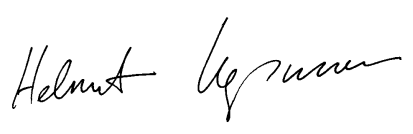

Prof. Dr. Helmut Messmann

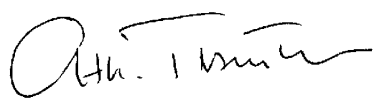

Prof. Dr. Christian Trautwein

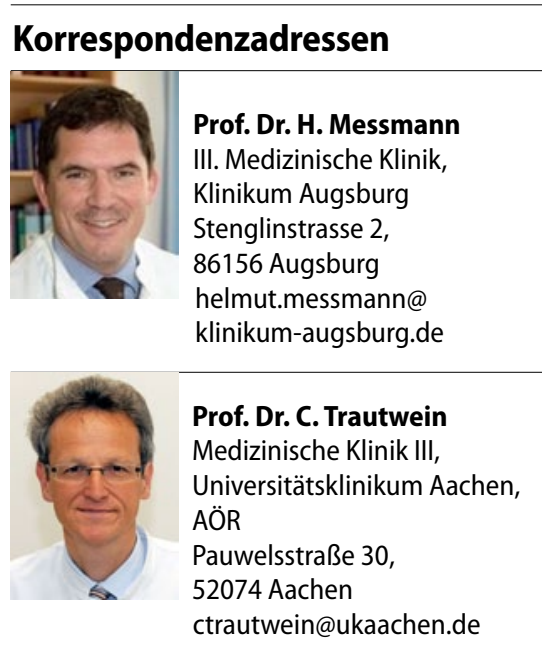

Interessenkonflikt. H. Messmann gibt an, dass kein Interessenkonflikt besteht. C. Trautwein hat Vortragshonorare von den Firmen Gilead, AbbVie, BMS, Roche, Falk und Astra Zeneca erhalten. 